\title{
Neuroenhancement - A Controversial Topic in Contemporary Medical Ethics
}

\author{
Kirsten Brukamp and Dominik Gross \\ Institute for History, Theory, and Ethics of Medicine, \\ RWTH Aachen University, \\ Germany
}

\section{Introduction}

Neuroenhancement will become an important topic of medical ethics in future years and decades, due to the increasing insights of neuroscience into the functions of the brain and the growing possibilities of meaningful interventions. Consequently, several crucial topics need to be discussed in order to address this emerging issue, and these topics correspond to the following sections of this article:

1. Introduction

2. Enhancement: How is enhancement defined? Which topics in the theory of medicine does it touch on, and which concepts help to understand it better?

3. Neuroenhancement and its categories: What is neuroenhancement in particular? What are the ends and methods of neuroenhancement? Which subtypes exist, and how can they be classified?

4. Pharmacological and technological neuroenhancement: Which medication is used for pharmacological neuroenhancement? How may technological advancements be utilized to achieve neuroenhancement?

5. Principles in medical ethics: How are ethical judgments usually made in medical ethics? According to which criteria should neuroenhancement be assessed in general?

6. Ethical assessment of neuroenhancement: Which arguments are used to support or criticize neuroenhancement? What are the advantages and disadvantages? How can neuroenhancement currently be assessed and judged?

7. Conclusion

\section{Enhancement}

Enhancement strategies in medicine, in general, are opposed to the classic framework of medical interventions - namely therapeutic, preventive, rehabilitative, and palliative measures -, in so far as no medical reasons, i.e. indications, for them exist: The people who request enhancements are not affected by disease according to classic standards and have not received a medical diagnosis related to their wishes. The intention behind enhancements is the improvement of subjective well-being and a higher quality of life. Although a general definition differentiates between therapy and enhancement, the dividing line needs to be 
outlined separately for each type of treatment. A "treatment" for enhancement purposes is not synonymous with "therapy" any longer. Examples for enhancement include aesthetic surgery, doping in sports, and the use of presumed anti-aging medication.

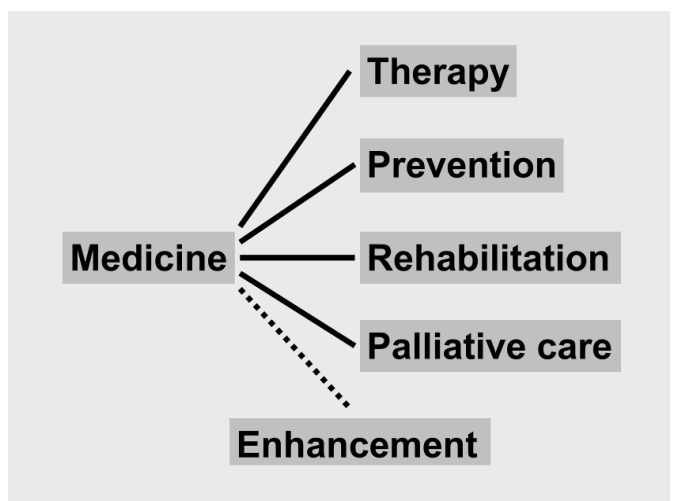

Fig. 1. Medicine comprises a multitude of different concepts for the wide notion of health care. Enhancement, however, is not usually considered a classic part of this, even though overlaps exist in expertise and methods.

The "enhancement" concept elicits a number of questions in the realm of the theory of medicine (Groß, 2011): What is a "disease" state, as opposed to "health"? Can a state of "normalcy" be defined? Are these views not culture-sensitive and changing throughout history? In addition, social and ethical concerns follow: In which way is the physicianpatient relationship going to change when more enhancement options become available? Is the "patient" concept in medicine replaced by a "client" concept?

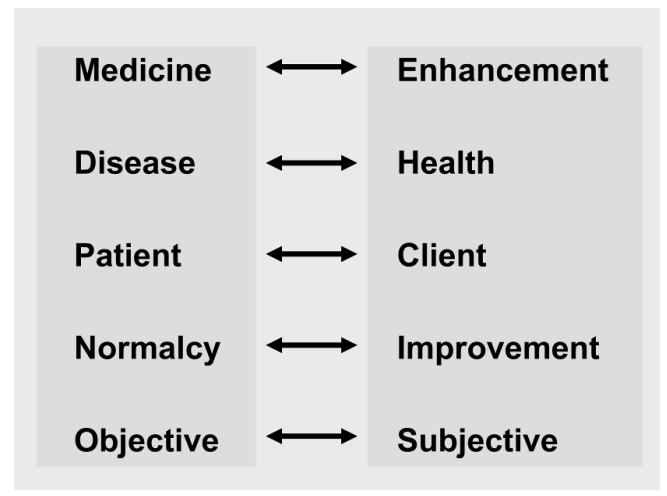

Fig. 2. The difference between medicine and enhancement may, in simplified form, be portrayed by antonyms, although the conceptual distinction is complex and possesses many implications, which still need to be pondered in the theory of medicine.

Distinguishing between therapy and enhancement is culture-dependent. The differences between them cannot be determined by science alone. Rather, it is the role of science to 
provide data about biological and medical facts. Based on this, societies then decide which functional states count as "normal", "variant", "different", or "diseased", just to name a few possibilities for overall assessments. These judgments are difficult to make, given the complexities that result from an interaction between phenotypical features that are problematic to classify individually themselves. Three specific examples deserve mention: complications of features, population biology, and life stages.

Complications of features: A population may possess a feature $\mathrm{M}$ in the majority of cases, with a complication rate of $\mathrm{X} \%$ for a serious health problem, which is undesirable according to public opinion. In addition, there may be a variant feature $\mathrm{V}$ in a minority, with a higher complication rate of $(X+Y) \%$ for the serious complication. How is social acceptance for feature $\mathrm{V}$ achieved on the individual or the population level? When does feature $\mathrm{V}$ become a disease? Does this depend on the percentage with which it occurs in the population, on the seriousness of the complication, or on the complication rate? These questions cannot be contemplated within science alone; the answers require a qualified insight into the practices, needs, and desires of a culture.

Population biology: From a population standpoint, e.g. in evolution, it is advantageous to produce a variability of features, within certain limits. This makes the population more stable in its niche. The features that are currently present have developed over many generations and are balanced with each other. Under these circumstances, simultaneous, congruent shifts in features can reduce adaptive capacities and may be detrimental for a population as a whole, although they could certainly be beneficial at times as well. When are such feature shifts healthy or abnormal? - In parallel, it is unclear what the sequelae will be when a significant number of humans want to change their appearances, skills, or habits in a certain direction at the same time through enhancement. From a societal perspective, it may be better to discourage such a desire.

Life stages: It remains unclear how to classify changes that occur relatively often during temporary stages of life only, for example childhood and puberty, pregnancy and old age. Is lower extremity edema during pregnancy normal or a disease? Is cognitive decline with increasing age normal, or is old age a disease with dementia as one of its signs? These puzzles illustrate that the dividing line between health and disease becomes blurry in special, but still fairly common situations. Sometimes, it is best to explain states in terms of their physiology and pathophysiology rather than to apply a biased term too early.

In summary, the concept of enhancement is vague because the notion of disease in medicine is debated as well. In addition, the intentions for enhancements are subjective: What counts as an improvement for one person may be unacceptable for another.

\section{Neuroenhancement and its categories}

Neuroenhancement is the use of enhancement strategies - according to the definition for enhancement in general - that affect the nervous system. More specifically, such a use, in the narrow sense, mostly concerns the central nervous system because there are limited potential applications in the peripheral nervous system. Classifications of neuroenhancement may be developed according to two distinct categories: 1 . the cognitive functions that are to be improved as well as the intentions and aims that underlie the use 
and 2. the methods or routes that are employed. The different methods may result in permanent or temporary enhancement.

Categories of neuroenhancement according to cognitive functions

1. Sensory perception

2. Motor action

3. Communication

4. Mood and emotions

5. Cognition, narrowly conceived (e.g. attention, memory, decision-making)

6. Social and moral behavior

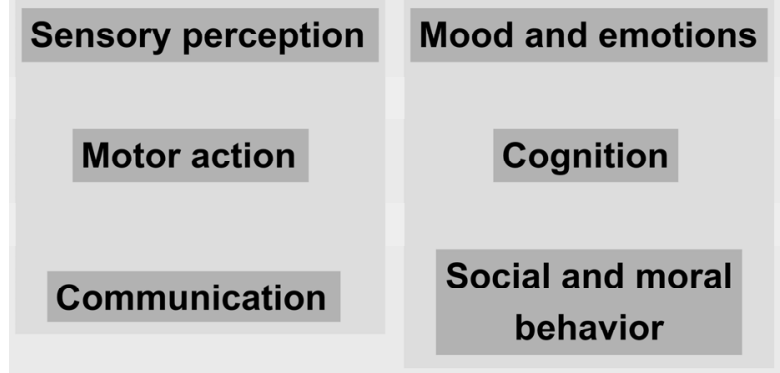

Fig. 3. Categories of neuroenhancement according to cognitive functions.

This classification by cognitive roles and intentions covers all major functions that the central nervous system usually exercises. One overarching aim is to improve the interaction between the subject and the environment: A human needs to communicate effectively with her surroundings; both sensory uptake and motor output help this purpose. The other central aim is to expand the overall behavioral repertoire. This allows the person to act more freely and gives her more options to choose from - cognitively, socially, and morally.

Neuroenhancement regarding sensory perception may mean to improve the functioning of the human senses or to potentially acquire new types of perception. For example, visual and auditory senses could be enhanced to include new wavelengths and frequencies outside the usual visible and audible spectrum. Regarding olfaction, humans may imitate dogs; as with all types of enhancement, however, it needs to be seen why they would want to procure this particular ability. - Motor neuroenhancement could lead to more efficient steering of technical devices. This type of use is largely futuristic; nevertheless, medical reports on tetraplegia (Hochberg et al., 2006) demonstrate that brain-machine interfaces can be utilized as therapies in order to expand the motor skills of severely compromised, wheelchair-bound patients. - Communication, enhanced by neurotechnology, appears as a promise with a high commercial potential. After all, recent years have seen a rise in communication technology, both towards large social networks and in favor of brief messages of an informational or emotional nature. Both trends could potentially continue with neuroenhancement.

Emotions and mood are typically improved for therapeutic purposes in depressive patients. The slogan "Feeling "better than well"' (Hall, 2004) seems to suggest that people who consider their emotions as normal may feel even happier, more euphoric, or generally enhanced when they take antidepressants. - Cognitive functions in the narrow sense, such as 
attention and memory, may become better through neuroenhancement as well. Some pharmaceutical drugs developed for medical purposes do improve concentration, at least temporarily (Repantis et al., 2010). Medication that reduces the need for sleep also belongs to this general category. So-called "memory chips" are still very much a matter of speculation: These devices would store information outside of the brain, and they would allow access to deposit and retrieve these data (Groß, 2009b). - Social neuroscience aims at elucidating the determinants of living in groups and cultures. Eventually, the scientific results could be translated into opening up options for favorable social and moral behavior.

A further classification of neuroenhancement relies on the methods that proponents pursue to achieve the goal.

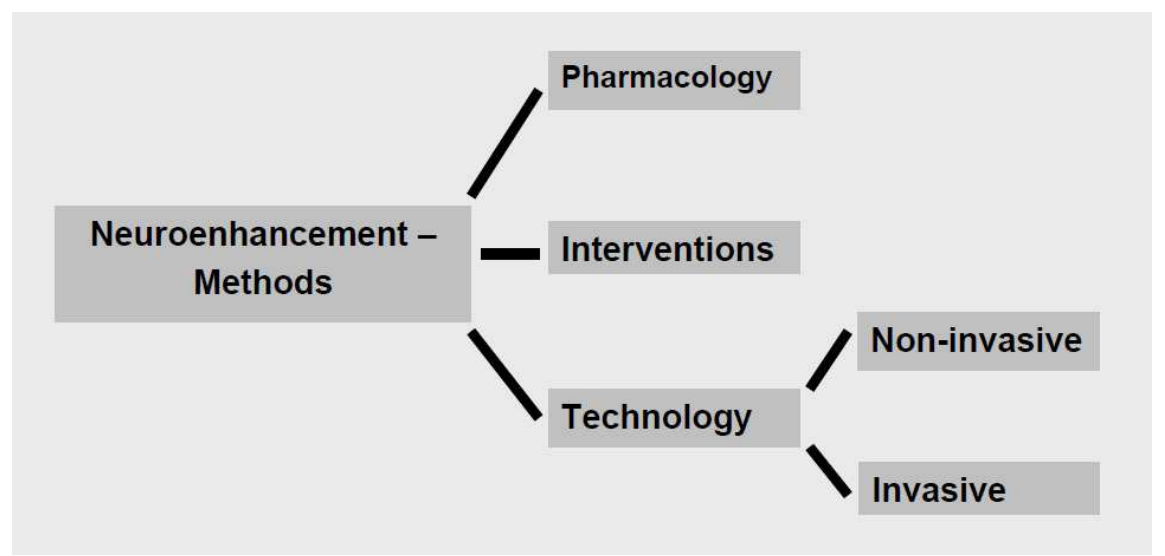

Fig. 4. Categories of neuroenhancement according to the methods employed.

Categories of neuroenhancement according to the methods employed

1. Pharmacology

2. Interventions (surgical or minimally invasive)

3. Non-invasive, external technology

4. Invasive, internal technology

In the following, the focus will be on pharmacological measures and implanted technology.

\section{Pharmacological and technological neuroenhancement}

Pharmacological neuroenhancement is known under different names, such as "cosmetic neurology" and "brain doping". These latter terms clearly reflect the enhancement character of the endeavor. The medications used belong to a variety of medical classes, including pharmaceutical drugs used against depression (e.g. fluoxetine), dementia (e.g. donepezil), attention-deficit hyperactivity disorder (e.g. methylphenidate), and narcolepsy and sleeping disorders (e.g. modafinil) (Groß, 2009a).

Neuroenhancing medication, therefore, is already a reality to a degree - as evidenced by the prevalence for the use of methylphenidate, modafinil, and antidepressants for this purpose particularly in the United States of America (Greely et al., 2008). The drugs are used in 
schools, at universities, and at work for similar purposes: to improve objective performance parameters and to boost self-esteem.

Interventions for neuroenhancement may be performed by microsurgery; however, these techniques do not yet exist. Non-invasive, external technology comprises brain stimulation techniques, such as transcranial magnetic stimulation (TMS) and transcranial direct current stimulation (tDCS). Although these methods possess potential to enhance brain function, they have not been developed to a degree to allow a reliable use for enhancement at the present time.

The technological advancements that support neuroenhancement are largely futuristic. As discussed above, they can be categorized into attempts to improve sensory, motor, communicative, emotional, cognitive, and social functions. The technological products that are already available suggest that some enhancement strategies may be successful more readily than others. In particular, the following applications come to mind: supersensory perception, prosthesis steering, and more efficient communication.

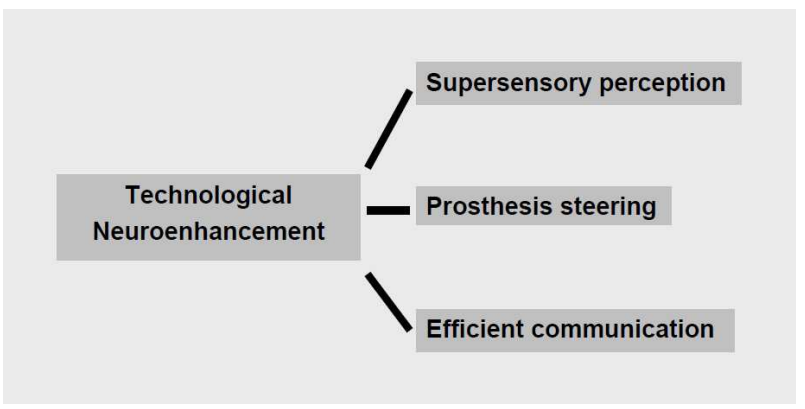

Fig. 5. Pursuits in technological neuroenhancement that are currently in development.

Supersensory perception is already on the horizon, given the medical treatments of retinal, cochlear, and auditory brainstem implants (Groß, 2007a). Both humans (Hochberg et al., 2006) and non-human primates (Velliste et al., 2008) have been capable of using brainmachine interfaces to steer prosthesis. Brain signals can be analyzed for motor-independent communication (Monti et al., 2010).

Emotions and mood may be changed by internal devices as well. In curative medicine, deep brain stimulation is an accepted treatment for Parkinson's disease and essential tremor as well as other diseases (Deep-Brain Stimulation for Parkinson's Disease Study Group, 2001). Deep brain stimulation is administered through electrodes that are implanted in deep regions of the brain, such as basal ganglia. Its effects include neurological and psychological ones, such as both positive and negative influences as well as side effects on extrapyramidal motor functions and emotional activation, which oftentimes manifests as euphoria and mania (Müller \& Christen, 2011). Accordingly, a mild mode of stimulation might improve mood without other detrimental side effects. At this time, however, a wide-spread use of rather large, invasive electrodes is not conceivable for enhancement purposes. Regarding cognitive functions, memory chips have been discussed, but continue to remain elusive for now. Social and moral behavior is so complex and adaptable that it is currently unclear how to specifically enhance it, outside of general effects on emotional and cognitive states. 
Neurotechnology has already been demonstrated to exhibit rather upsetting effects in some cases. In rats, a selective microstimulation technology for select brain areas has been used to coerce the animals into following certain paths that they would otherwise have shied away from (Talwar et al., 2006). Such applications lead to criticism against brain interventions per se.

\section{Principles in medical ethics}

Neuroenhancement can be assessed according to standard approaches in medical ethics, e.g. principlism, a strong contemporary attitude in medical ethics. It takes into consideration four principles, which draw widespread approval even from divergent ideological positions, namely autonomy, beneficence, non-maleficence, and justice (Beauchamp \& Childress, 2009). These four tenets of the principlism approach in medical ethics appear to be very well applicable to emerging neurotechnology (Brukamp, 2010).

The individual patient exercises his right to autonomy. Beneficence and non-maleficence are insofar related as they both stem from the perspective of an external, caring entity that seeks to minimize harm and maximize benefit to the individual. Justice is oftentimes applied from a standpoint above the individual, from a level of society, where all goods are supposed to be distributed according to just principles.

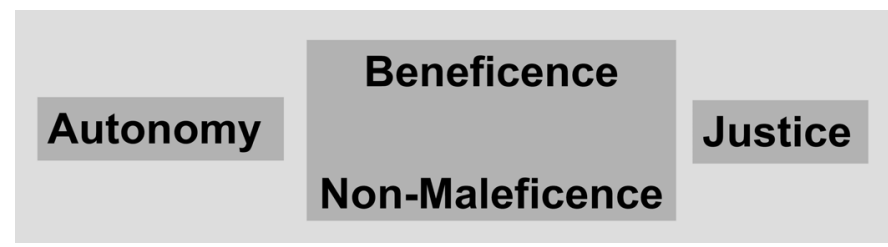

Fig. 6. The four principles in the principlism approach within medical ethics.

The principle of autonomy suggests enabling patients to exercise independence in their decision-making. They have to give their informed consent to any medical measure: First, patients are supposed to receive information about their conditions and treatment options, and this information needs to be tailored to their level of understanding. Second, they are to weigh the possibilities themselves and decide about the course of action that suits them best.

Beneficence in the medical realm means to look out for and foster the benefit of patients or subjects. It may encompass supporting the development of devices to aid humans and acquiring evidence for their efficiency. Obviously, beneficence implies that technology, which helps humans, is backed in a number of ways, be it concerning research, development, production, accessibility, and distribution. Medical devices also need to be tested for efficiency according to the approved standards of evidence-based medicine (Sackett et al., 1996). Also, therapies are becoming more and more personalized, a trend that will likely grow in the future. This way, the therapy and enhancement strategies best suited for patients and subjects can be chosen out of a whole array of possibilities.

Non-maleficence, as a principle in medical ethics, asks for a relative absence of negative side effects in relationship to the expected benefits. For example, a treatment with fewer side effects is preferable to one with more when both have the same efficacy. The traditional gold standard is an important guideline for comparison. The side effects need to be assessed in 
relation to the benefits: Patients may prefer therapies that improve survival with more extensive side effects to those that only work on symptoms with less side effects.

Distributive justice means that there is a kind of justified distribution of goods in society. This principle requires competent applications to specific situations. Theories of justice are wide, varied, and heterogeneous, and it therefore defies a brief, comprehensive discussion.

Enhancement partially transcends medicine and is therefore subject to a distinct assessment. Nevertheless, enhancement falls under the extended realm of medical ethics so that the principlism approach still applies:

First, neuroenhancement concerns the human being as a biological entity. It alters the physiological functions to achieve something unknown and unprecedented. Therefore, those principles from medical ethics apply that are relevant for the human as a biological entity.

Second, the experts, who assist in the transformation, are the same in medical treatment and medical enhancement: For example, physicians' expertise is needed to safely develop and apply new medications, interventions, and technologies. Since enhancement falls under the responsibility of medical providers, the normative principles from medical law and medical ethics apply by extension.

Accordingly, applying the four principles from medical ethics yields the following perspectives and arguments, among others (Brukamp, 2010):

Beneficence and non-maleficence may help to decide between different categories of neuroenhancement, namely pharmacological and technological ones. These strategies have diverse side effect profiles. Local treatments, instead of pharmacological ones, may sometimes meanless systemic side effects for the body. Conversely, pharmacological treatments can better be titrated, and increasing knowledge in cell and molecular biology might enable greater insights into mechanisms and eventually more specific treatments with medication. The number of options makes it possible to personalize the enhancement to individual needs. As a general rule, non-invasive measures are preferable to invasive ones because of a more moderate side effect profile.

The steering of technological aids by neural signals, as one example of a potential future application in both neuromedicine and neuroenhancement, requires the acquisition of large amounts of digital data. According to the value of privacy, backed by the principles of autonomy, beneficence, and non-maleficence, such data should be utilized only for the benefit of the patients with their informed consent. Confidentiality in this context extends not only to the medical personnel, but to all people who come into contact with the data. This tenet becomes more difficult to be reinforced outside the medical field proper: Confidentiality has a tradition in professional contexts such as medicine, psychology, law, and theology, but its implications are less well known to other fields and the general public.

The principle of justice entails that financial, time, personnel, and other resources in the health care system should not be diverted away from those who deserve them most. Therapies were developed to help the sick, disabled, and disadvantaged. Therefore, it would be wrong to strain the infrastructure and the resources of the medical system with the desires of too many clients who ask for enhancements. 


\section{Ethical assessment of neuroenhancement}

Applying the prominent four principles (Beauchamp \& Childress, 2009) is one approach of addressing problems in medical ethics. Nevertheless, pro and con arguments may alternatively and more conveniently be grouped according to central topics, which seem to be defining features for the field in question. Accordingly, the current problems of pharmacological neuroenhancement, which extend, in part, to all types of potential future neuroenhancement, can be discussed along the issues of medical risk, lack of evidence, human nature, and justice.

\section{Risk Nature \\ Evidence \\ Justice}

Fig. 7. For an overall assessment of neuroenhancement, there should be, among others, a consideration of medical risk, medical evidence, human nature, and distributive justice. The former two still belong to the narrower realm of responsibility for the field of medicine, whereas the arguments regarding the latter are derived from philosophical theory.

Pharmacological neuroenhancers carry several medical risks with them (Banjo et al., 2010): They have side effects, there is a risk of addiction, and they may lose efficacy over time so that higher doses or drug combinations lead to even more risks. In addition, the medications may result in a wrongful subjective overestimation of one's abilities, e.g. regarding attention and insomnia while driving in traffic, which can endanger oneself and others. In most cases, the long-term effects in the healthy population have simply not been studied and are unknown.

The scientific and medical evidence that pharmacological neuroenhancers reliably work as expected is scarce. Reviews of the available data show that methylphenidate and modafinil can be said to exhibit some desired effects on healthy people, but may also lead to dangerous overconfidence in one's possibilities (Repantis et al., 2010). Importantly, the end points for potential studies that test neuroenhancers have not been adequately defined: Which ones would be truly deserving and valid end points? They should extend beyond short-term effects and be compatible with other goals that humans have in life. Once these end points are defined, high-quality studies then need to prove the efficacy of the neuroenhancers to achieve them, according to the standards of evidence-based medicine (Sackett et al., 1996).

Humans normally value truth, authenticity, and personal identity (The President's Council on Bioethics, 2003). Some opponents to neuroenhancement regard artificial means for enhancements as (self-)deception and as a threat to human nature. Nevertheless, humans have always tried to use tools and to embellish themselves by socially accepted external means - this seems to be a feature of human nature itself. Consequently, the reference to human nature is not one that helps to decide easily between appropriate and inappropriate 
means of self-improvement; rather, it needs to be applied aptly to different contexts (Groß, 2007a, 2007b).

Supporters of neuroenhancement frequently argue that behavioral modifications have always been employed to achieve the same goals that neuroenhancement serves (Greely et al., 2008) - for example, measures like classic learning and psychotherapy have widely been accepted as valid means. These proponents do not admit that a clear-cut boundary exists between the traditional methods for self-improvement and the novel prospects of neuroenhancement. Nevertheless, such distinctive features of the latter are indeed present and discernible: Medical risks are higher, due to more direct alterations in the brain by medication use or invasive measures; procedures deemed artificial can result in feelings of external determination and self-alienation; effects may suddenly cease secondary to a lack of access to medication or because of technological malfunction, which may be immediate and unforeseeable phenomena.

Considerations from justice concern access and coercion. While neuroenhancement may serve the purpose of creating a level playing field for naturally disadvantaged people, performance gaps between groups may widen because of unequal access to the supposedly enhancing medication. Moreover, vulnerable groups, such as children, could experience peer pressure in favor of drug use. Due to their vulnerability, for example because of ongoing brain development, the negative side effects may actually become more extensive.

In summary, a number of arguments caution against the use of neuroenhancement, in particular of the pharmacological variety in use today. Medical arguments refer to risks from medication side effects and from overestimations of one's own abilities, which may become dangerous for oneself and others. Besides, the current evidence for the efficacy of neuroenhancers, aside from short-term effects of some, is thin. While human beings possess a desire for enhancements, not all types are considered compatible with the development and protection of human nature and identity. Enhancement in general may divert resources away from the classic medical system, constituting a challenge to the principle of distributive justice (Racine \& Forlini, 2009). Sociological arguments claim that equal access to enhancement routes cannot be guaranteed, and coercion may result in ill effects, particularly in vulnerable groups who cannot decide for themselves, like children.

\section{Conclusion}

In conclusion, neuroenhancement poses problems due to its nature as a novel enhancement method and because it concerns an organ that mediates human identity. While it cannot be ruled out that acceptable and affordable means may be developed in the future, the longterm benefits cannot easily be foreseen at this time, both on the individual level and from the perspective of society as a whole. Therefore, the best advice is a cautious approach, and the current situation warrants an overall precautionary stance towards neuroenhancement.

\section{Acknowledgements}

This paper was in part supported by the project "AC-TEC: Gender-Related Acceptance, Usability, and Ethics in New (Medical) Technologies" of the "Exploratory Research 
Space@Aachen (ERS)" as part of the German "Exzellenzinitiative des Bundes und der Länder" ("Excellence Initiative"), funded by the "Deutsche Forschungsgemeinschaft (DFG)" ("German Research Foundation").

\section{References}

Banjo, O.C.; Nadler, R., \& Reiner, P.B. (2010). Physician attitudes towards pharmacological cognitive enhancement: safety concerns are paramount. PLoS ONE Vol. 5 No. 12, pp. e14322

Beauchamp, T.L., \& Childress, J.F. (2009). Principles of biomedical ethics, Oxford University Press, New York / Oxford

Brukamp, K. (2010). Aspekte zur medizinethischen Beurteilung von inkorporierter Technologie im Bereich des Gehirns [Aspects of medical ethics to assess internal technology in the brain], In: Akzeptanz, Nutzungsbarrieren und ethische Implikationen neuer Medizintechnologien: die Anwendungsfelder Telemedizin und Inkorporierte Technik. [Acceptance, barriers to use, and ethical implications of new medical technologies: applications in telemedicine and internal techniques], Groß, D.; Gründer, G., \& Simonovic, V. (Eds.), pp. 119-124, Kassel University Press, Kassel

Deep-Brain Stimulation for Parkinson's Disease Study Group. (2001). Deep-brain stimulation of the subthalamic nucleus or the pars interna of the globus pallidus in Parkinson's disease. New England Journal of Medicine, Vol. 345, No. 13, pp. 956-963

Greely, H.; Sahakian, B.; Harris, J.; Kessler, R.C.; Gazzaniga, M.; Campbell, P., \& Farah, M.J. (2008). Towards responsible use of cognitive-enhancing drugs by the healthy. Nature Vol. 456, pp. 702-705

Groß, D. (2007a). Neurobionisches und psychopharmakologisches Enhancement - Teil 1: Definitionen, Einsatzbereiche und gesellschaftliche (Vor-)Urteile [Neurobionic and psychopharmacological enhancement - part 1: definitions, areas of use, and judgments and biases in society], In: Sind die Gedanken frei? Die Neurowissenschaften in Geschichte und Gegenwart [Are thoughts free? The neurosciences in history and present], Groß, D., \& Müller, S. (Eds.), pp. 216-241, Medizinisch Wissenschaftliche Verlagsgesellschaft, Berlin

Groß, D. (2007b). Neurobionisches und psychopharmakologisches Enhancement - Teil 2: Medizinethische Anmerkungen zu einer aktuellen Debatte [Neurobionic and psychopharmacological enhancement - part 2: remarks from medical ethics on a contemporary debate], In: Sind die Gedanken frei? Die Neurowissenschaften in Geschichte und Gegenwart [Are thoughts free? The neurosciences in history and present], Groß, D., \& Müller, S. (Eds.), pp. 242-252, Medizinisch Wissenschaftliche Verlagsgesellschaft, Berlin

Groß, D. (2009a). Blessing or curse? Neurocognitive enhancement by "brain engineering". Medicine Studies Vol. 1, No. 4, pp. 379-391

Groß, D. (2009b). Neurobionik und neurobionisches Enhancement - Eine Technik der Zukunft? [Neurobionics and neurobionic enhancement - a technique of the future?], In: Chancen und Risiken der Neurowissenschaften [Chances and risks of the neurosciences], Müller, S.; Zaracko, A.; Groß, D., \& Schmitz, D. (Eds.), pp. 74-78, Lehmanns Media, Berlin

Groß, D. (2011). Traditional vs. modern neuroenhancement: notes from a medico-ethical and societal perspective, In: Implanted minds: the neuroethics of intracerebral stem cell 
transplantation and deep brain stimulation, Fangerau, H.; Fegert, J.M, \& Trapp, T. (Eds.), pp. 291-311, Transcript, Bielefeld

Hall, W. (2006). Feeling "better than well“. EMBO Reports Vol. 5, pp. 1105-1109

Hochberg, L.R.; Serruya, M.D.; Friehs, G.M.; Mukand, J.A.; Saleh, M.; Caplan, A.H.; Branner, A.; Chen, D.; Penn, R.D., \& Donoghue, J.P. (2006). Neuronal ensemble control of prosthetic devices by a human with tetraplegia. Nature Vol. 442, No. 7099, pp. 164171

Monti, M.M.; Vanhaudenhuyse, A.; Coleman, M.R.; Boly, M.; Pickard, J.D.; Tshibanda, L; Owen, A.M., \& Laureys, S. (2010). Willful modulation of brain activity in disorders of consciousness. New England Journal of Medicine Vol. 362, No. 7, pp. 579-589

Müller, S., \& Christen, M. (2011). Deep brain stimulation in Parkinsonian patients - Ethical evaluation of cognitive, affective, and behavioral sequelae. AJOB Neuroscience Vol. 2, No. 1, pp. 3-13

Racine, E., \& Forlini, C. (2009). Expectations regarding cognitive enhancement create substantial challenges. Journal of Medical Ethics Vol. 35, pp. 469-470

Repantis, D.; Schlattmann, P.; Laisney, O., \& Heuser, I. (2010). Modafinil and methylphenidate for neuroenhancement in healthy individuals: a systematic review. Pharmacological Research Vol. 62, No. 3, pp. 187-206

Sackett, D.L.; Rosenberg, W.M.; Gray, J.A.; Haynes, R.B., \& Richardson, W.S. (1996). Evidence-based medicine: what it is and what it isn't. British Medical Journal Vol. 312, No. 7023, pp. 71-72

Talwar, S.K.; Xu, S.; Hawley, E.S.; Weiss, S.A.; Moxon, K.A., \& Chapin, J.K. (2002). Rat navigation guided by remote control. Nature Vol. 417, No. 6884, pp. 37-38

The President's Council on Bioethics (2003). Beyond therapy: biotechnology and the pursuit of happiness, Washington, DC

Velliste, M.; Perel, S.; Spalding, M.C.; Whitford, A.S., \& Schwartz, A.B. (2008). Cortical control of a prosthetic arm for self-feeding. Nature Vol. 453, No. 7198, pp. 10981101. 


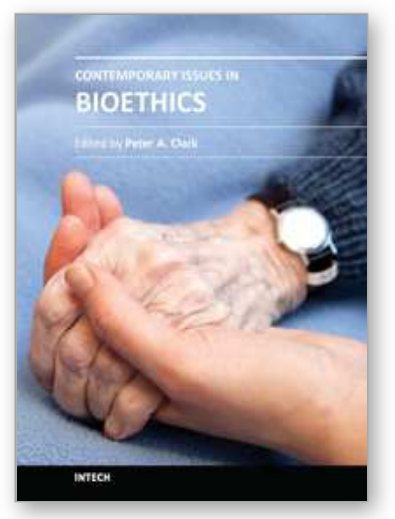

\section{Contemporary Issues in Bioethics}

Edited by Dr. Peter A. Clark

ISBN 978-953-51-0169-7

Hard cover, 162 pages

Publisher InTech

Published online 07, March, 2012

Published in print edition March, 2012

The main strength of this book is the international exchange of ideas that will not only highlight many of these crucial bioethical issues but will strengthen the discipline of bioethics both nationally and globally. A critical exchange of ideas allows everyone to learn and benefit from the insights gained through others experiences. Analyzing and understanding real medical-ethical issues and cases and how they are resolved is the basis of education in bioethics for those who will have to make these decisions in the future. The more we examine, analyze, and debate these bioethical issues and cases, the more knowledge will be gained and hopefully, we will all gain more practical wisdom.

\section{How to reference}

In order to correctly reference this scholarly work, feel free to copy and paste the following:

Kirsten Brukamp and Dominik Gross (2012). Neuroenhancement - A Controversial Topic in Contemporary Medical Ethics, Contemporary Issues in Bioethics, Dr. Peter A. Clark (Ed.), ISBN: 978-953-51-0169-7, InTech, Available from: http://www.intechopen.com/books/contemporary-issues-in-bioethics/neuroenhancement-acontroversial-topic-in-medical-ethics

\section{INTECH}

open science | open minds

\section{InTech Europe}

University Campus STeP Ri Slavka Krautzeka 83/A 51000 Rijeka, Croatia

Phone: +385 (51) 770447

Fax: +385 (51) 686166 www.intechopen.com

\section{InTech China}

Unit 405, Office Block, Hotel Equatorial Shanghai No.65, Yan An Road (West), Shanghai, 200040, China 中国上海市延安西路65号上海国际贵都大饭店办公楼 405 单元 Phone: +86-21-62489820

Fax: $+86-21-62489821$ 
(C) 2012 The Author(s). Licensee IntechOpen. This is an open access article distributed under the terms of the Creative Commons Attribution 3.0 License, which permits unrestricted use, distribution, and reproduction in any medium, provided the original work is properly cited. 